\title{
The resonance peak in cuprate superconductors
}

\author{
Dirk K. Morr ${ }^{a}$ and David Pines ${ }^{a, b}$ \\ ${ }^{a}$ University of Illinois at Urbana-Champaign, Loomis Laboratory of Physics, 1110 W. Green St., Urbana, Il, 61801 \\ ${ }^{b}$ Center for Materials Science, LANSCE, and Center for Nonlinear Studies, Los Alamos National Laboratory, Los Alamos, \\ $N M 87545$
}

(August 13, 2018)

\begin{abstract}
We pursue the consequences of a theory in which the resonance peak observed in inelastic neutron scattering (INS) experiments on underdoped and optimally doped $\mathrm{YBa}_{2} \mathrm{Cu}_{3} \mathrm{O}_{6+x}$ compounds arises from a spin-wave excitation. We find that it is heavily damped, and thus almost not observable, in the normal state, but becomes visible in the superconducting state due to the drastic decrease in spin damping. We show that a spin-fermion model correctly describes the temperature dependence of the peak position for $\mathrm{YBa}_{2} \mathrm{Cu}_{3} \mathrm{O}_{7}$, as well as the doping dependence of the peak position and of the integrated intensity. We explain why no resonance peak has been observed in $\mathrm{La}_{2-x} \mathrm{Sr}_{x} \mathrm{CuO}_{4}$, and make several predictions concerning resonance peaks in other cuprate superconductors.
\end{abstract}

Recent inelastic neutron scattering (INS) experiments have shown that the sharp magnetic collective mode ("resonance peak"), which was first observed in the superconducting state of $\mathrm{YBa}_{2} \mathrm{Cu}_{3} \mathrm{O}_{7}$ [1] and only appears in the odd channel, also exists in the underdoped $\mathrm{YBa}_{2} \mathrm{Cu}_{3} \mathrm{O}_{6+x}$ compounds [2 7]. Several groups find that as the doping decreases $\omega_{\text {res }}$, the peak frequency decreases [2 77, while both, the peak width in frequency space and its integrated intensity increase [4,5]. In the underdoped systems, a considerably broadened peak at $\omega_{\text {res }}$ is also observed in the normal state [4, 6]. Fong et $a l$. also find that $\omega_{\text {res }}$ shifts to higher frequencies with decreasing temperature in the superconducting state of $\mathrm{YBa}_{2} \mathrm{Cu}_{3} \mathrm{O}_{7}$ [8].

These new results put tight restrictions on the theoretical scenarios proposed after the discovery of the resonance peak in $\mathrm{YBa}_{2} \mathrm{Cu}_{3} \mathrm{O}_{7}$; these ascribed the resonance peak to a final state interaction between the fermionic particles [9], band structure anomalies [10], interlayer tunneling [11], a new collective mode in the particleparticle channel [12], or a collective spin-wave mode brought about by strong antiferromagnetic correlations [13]. In particular, the observation that $\omega_{\text {res }}$ decreases with decreasing doping, while the superconducting gap $\Delta_{S C}$ is approximately constant, as seen in ARPES 14] and tunneling experiments [15] on Bi2212, contradicts scenarios in which $\omega_{\text {res }} \approx 2 \Delta_{S C}$ [9 11 .

In this communication we use the spin-fermion model to show that the spin-wave scenario, perhaps uniquely, provides a natural explanation for all above-cited experimental results. The dispersion of the spin-wave mode is

$$
\omega_{q}^{2}=\Delta_{s w}^{2}+c_{s w}^{2}(\mathbf{q}-\mathbf{Q})^{2},
$$

where $\Delta_{s w}$ is the spin-wave gap, $c_{s w}$ is the spin-wave velocity, $\mathbf{Q}=(\pi, \pi)$; the mode is damped due to its coupling to planar quasi-particles. In the superconducting state, where the spin damping is minimal, the resonance peak should always be observable provided $\Delta_{s w}<\omega_{c} \approx 2 \Delta_{S C}[16]$. For underdoped systems, at temperatures such that ARPES 14 experiment show a leading edge gap in the quasiparticle spectrum, the spin damping present at higher temperatures is reduced sufficiently that the resonance mode becomes visible. For optimally doped systems with no leading edge gap, the spin mode is overdamped and invisible. In contrast to the results of Refs. [9 11], the existence of the resonance peak is not related to the interlayer coupling, and should be observed in single-layer compounds if the superconducting gap is large enough. We consider first the twolayer system $\mathrm{YBa}_{2} \mathrm{Cu}_{3} \mathrm{O}_{6+x}$, for which the bonding and anti-bonding tight-binding quasi-particle bands are given by

$$
\begin{aligned}
\epsilon_{\mathbf{k}}^{ \pm}= & -2 t\left(\cos \left(k_{x}\right)+\cos \left(k_{y}\right)\right) \\
& -4 t^{\prime} \cos \left(k_{x}\right) \cos \left(k_{y}\right) \pm t_{\perp}-\mu,
\end{aligned}
$$

where $t, t^{\prime}$ are the hopping elements between in-plane nearest and next-nearest neighbors, respectively, $t_{\perp}$ is the hopping between nearest-neighbors on different planes, and $\mu$ is the chemical potential. In a spin-fermion model [17], the spin-wave propagator, $\chi$, is given by

$$
\chi^{-1}=\chi_{0}^{-1}-\operatorname{Re} \Pi-i \operatorname{Im} \Pi,
$$

where $\chi_{0}$ is the bare propagator, and $\Pi$ is the irreducible particle-hole bubble.

Since the form of the bare propagator in Eq.(3) is model dependent, and thus somewhat arbitrary, we choose a form for $\left(\chi_{0}^{-1}-\operatorname{Re} \Pi\right)$ which is the lowest order expansion in momentum and frequency of a hydrodynamical form of $\operatorname{Re} \chi^{-1}$ [18 and can be shown to reproduce the INS experiments in the normal state of the underdoped $\mathrm{YBa}_{2} \mathrm{Cu}_{3} \mathrm{O}_{6+x}$ compounds [7, 19],

$$
\chi_{0}^{-1}-\operatorname{Re} \Pi=\frac{1+\xi^{2}(\mathbf{q}-\mathbf{Q})^{2}-\omega^{2} / \Delta_{s w}^{2}}{\alpha \xi^{2}},
$$

where $\xi$ is the magnetic correlation length, $\Delta_{s w}=c_{s w} / \xi$, and $\alpha$ is an overall constant. We assume that the form 
of Eq.(4) does not change in the superconducting state. Although Dai et al. [3] have recently found that for frequencies well below the resonance peak, the peaks in the spin fluctuation spectrum occur at incommensurate positions, which roughly scale with doping, we argue that the use of the commensurate form, Eq.(4), is justified for the description of the resonance peak since $(a)$ no resonance peak has been observed at incommensurate positions, $(b)$ incommensurate structure and the resonance peak are well separated in frequency, and $(c)$ the use of a commensurate instead of an incommensurate form will only influence low frequency results in the normal state.

With these assumptions, we need only calculate the imaginary part of $\Pi$ which describes the damping brought about by the decay of a spin excitation into a particlehole pair. In the odd channel, Im $\Pi$ only includes quasi-particle excitations between the bonding and antibonding bands. In the superconducting state we find to lowest order in the spin-fermion coupling $g_{\text {eff }}($ for $\omega>0$ )

$$
\begin{aligned}
\operatorname{Im} & \Pi_{\text {odd }}=\frac{3 \pi g_{e f f}^{2}}{8} \sum_{\mathbf{k}}\left(1-n_{F}\left(E_{\mathbf{k}+\mathbf{q}}^{+}\right)-n_{F}\left(E_{\mathbf{k}}^{-}\right)\right) \\
& \times\left[1-\frac{\epsilon_{\mathbf{k}+\mathbf{q}}^{+} \epsilon_{\mathbf{k}}^{-}+\Delta_{\mathbf{k}+\mathbf{q}} \Delta_{\mathbf{k}}}{E_{\mathbf{k}+\mathbf{q}}^{+} E_{\mathbf{k}}^{-}}\right] \delta\left(\omega-E_{\mathbf{k}}^{-}-E_{\mathbf{k}+\mathbf{q}}^{+}\right) \\
& +\left(n_{F}\left(E_{\mathbf{k}}^{-}\right)-n_{F}\left(E_{\mathbf{k}+\mathbf{q}}^{+}\right)\right)\left[1+\frac{\epsilon_{\mathbf{k}+\mathbf{q}}^{+} \epsilon_{\mathbf{k}}^{-}+\Delta_{\mathbf{k}+\mathbf{q}} \Delta_{\mathbf{k}}}{E_{\mathbf{k}+\mathbf{q}}^{+} E_{\mathbf{k}}^{-}}\right] \\
& \times\left\{\delta\left(\omega+E_{\mathbf{k}}^{-}-E_{\mathbf{k}+\mathbf{q}}^{+}\right)-\delta\left(\omega-E_{\mathbf{k}}^{-}+E_{\mathbf{k}+\mathbf{q}}^{+}\right)\right\},
\end{aligned}
$$

where $n_{F}$ is the Fermi function, and

$$
E_{\mathbf{k}}^{ \pm}=\sqrt{\left(\epsilon_{\mathbf{k}}^{ \pm}\right)^{2}+\left|\Delta_{\mathbf{k}}\right|^{2}}
$$

is the dispersion of the bonding and antibonding bands in the superconducting state, which we assume is described by the d-wave gap

$$
\Delta_{\mathbf{k}}=\Delta_{S C} \frac{\cos \left(k_{x}\right)-\cos \left(k_{y}\right)}{2} .
$$

The normal state result for $\operatorname{Im} \Pi_{\text {odd }}$ is recovered with $\Delta_{S C}=0$. We determine $g_{\text {eff }}$ by requiring that $\operatorname{Im} \Pi_{\text {odd }}$ reproduces the spin-damping seen at low frequencies in NMR experiments in the normal state of $\mathrm{YBa}_{2} \mathrm{Cu}_{3} \mathrm{O}_{6+x}$ [20] and assume that $g_{\text {eff }}$ does not change in the superconducting state.

We first discuss our results for $\mathrm{YBa}_{2} \mathrm{Cu}_{3} \mathrm{O}_{7}$, which were obtained with the following parameters: $t=300$ $\mathrm{meV}, t^{\prime}=-0.40 t, t_{\perp}=0.3 t, \mu=-1.27 t$ (which corresponds to a $22 \%$ hole concentration in the planes), and $\Delta_{S C}(T=0) \approx 25 \mathrm{meV}$, a value extracted from the tunneling experiments of Maggio-Aprile et al. [21]. In Fig. 1 we present our calculated result for $\operatorname{Im} \Pi_{o d d}$ at $\mathbf{Q}=(\pi, \pi)$ as a function of frequency for the normal (solid line) and superconducting state (dashed line). In the normal state the spin-damping increase linearly with

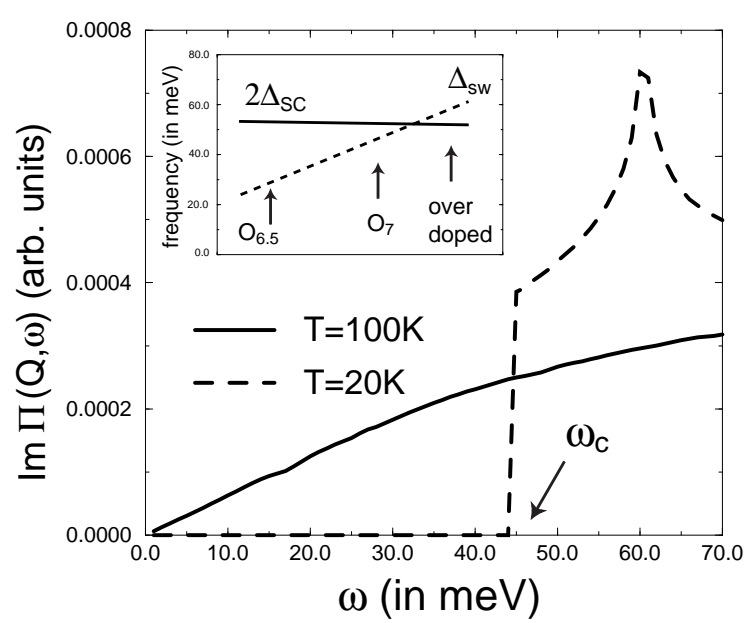

FIG. 1. $\quad \operatorname{Im}_{\text {odd }}$ at $\mathbf{Q}=(\pi, \pi)$ as a function of frequency in the normal state (solid line) and the superconducting state (dashed line). Inset: Schematic doping dependence of $\Delta_{s w}$ and $2 \Delta_{S C}$.

frequency, as is to be expected [22]. The spin-damping in the superconducting state is characterized by a step-like feature at $\omega_{c} \approx 2 \Delta_{S C}$ which arises from the creation of a particle-particle pair above the superconducting gap. At $T=0$, there are no quasi-particle excitations below $\omega_{c}$, and the spin-damping vanishes. The step in $\operatorname{Im} \Pi$ will then, via the Kramers-Kronig relation, lead to a logarithmic divergence in Re $\Pi$. This is neglected in Eq.(何), since the inclusion of fermion lifetime effects as found, e.g., in strong coupling scenarios, eliminates both, the sharp step in $\operatorname{Im} \Pi$ and the divergence in $\operatorname{Re} \Pi$.

In Fig. 2 we present our results for $\chi_{\text {odd }}^{\prime \prime}$ at $\mathbf{Q}=(\pi, \pi)$ as a function of frequency for the normal (solid line) and superconducting state (dashed line). In the normal state, the spin excitations are clearly overdamped and $\chi^{\prime \prime}$ exhibits a flat maximum around $\omega=20 \mathrm{meV}$. In contrast, in the superconducting state, the spin-damping is strongly reduced so that the spin-wave mode, which is now very sharp in frequency, becomes visible at $\omega_{\text {res }}=$ $\Delta_{s w}$, which we have chosen to be $41 \mathrm{meV}$ to reproduce the experimentally measured peak. The resulting spinwave velocity, $c_{s w}=\xi \Delta_{s w} \approx 90 \mathrm{meV}$ is quite reasonable, since $c_{s w}=(120 \pm 20) \mathrm{meV}$ in $\mathrm{YBa}_{2} \mathrm{Cu}_{3} \mathrm{O}_{6.5}$ and $c_{s w}=180 \mathrm{meV}$ in the undoped compound [7]. Our results can be easily understood using Eq.(4), since the intensity at $\omega=\Delta_{s w}$ is given by

$$
\chi_{o d d}^{\prime \prime}\left(\mathbf{Q}, \omega=\Delta_{s w}\right)=\left\{\operatorname{Im} \Pi_{o d d}\left(\mathbf{Q}, \Delta_{s w}\right)\right\}^{-1} .
$$

If $\Delta_{s w}<\omega_{c}, \operatorname{Im} \Pi_{o d d}$ in the superconducting state is much smaller than in the normal state and consequently, $\chi_{o d d}^{\prime \prime}$ is strongly enhanced at $\omega=\Delta_{s w}$. Our result is robust against changes in the band parameters, in contrast to those scenarios [9 11], which require a fine-tuning of parameters to observe a resonance peak. 


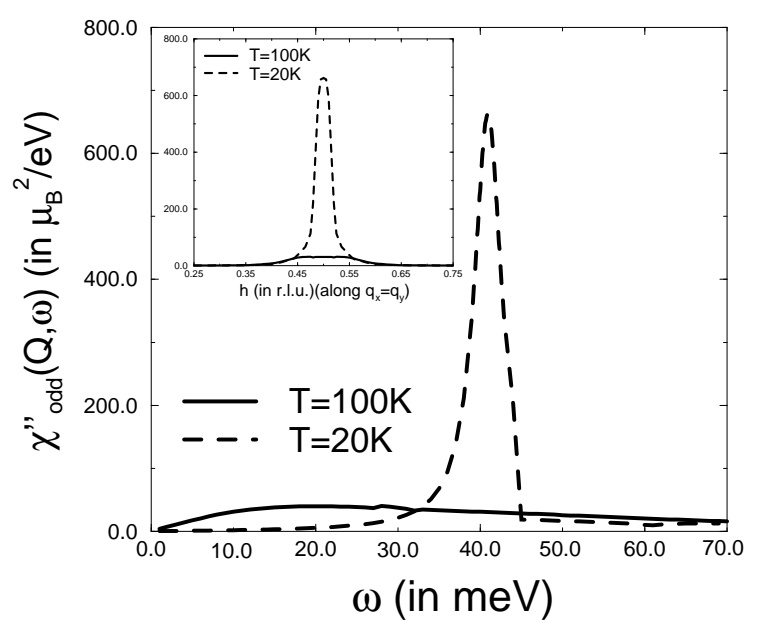

FIG. 2. $\quad \chi^{\prime \prime}$ at $\mathbf{Q}=(\pi, \pi)$ as a function of frequency in the normal state (solid line) and the superconducting state (dashed line). Inset: $\chi^{\prime \prime}$ for fixed frequency $\omega=41 \mathrm{meV}$ along $q_{x}=q_{y}$.

In the inset in Fig. 2 we plot $\chi_{o d d}^{\prime \prime}$ for fixed frequency $\omega=41 \mathrm{meV}$ along the $(0,0)$ to $(2 \pi, 2 \pi)$ direction in the normal (solid line) and superconducting state (dashed line). We find that the resonance peak is sharp in momentum space, in agreement with the experimental observations [3, 8]. The sharpness of the resonance peak in both momentum and frequency space can be easily understood from Eqs.(1D) and (4). One expects to find a resonance peak at $\omega_{q}$ which follows the dispersion of the spin excitations, Eq.(1) , as long as $\omega_{q}<2 \Delta_{S C}$. Since in $\mathrm{YBa}_{2} \mathrm{Cu}_{3} \mathrm{O}_{7}, \Delta_{s w} \approx 2 \Delta_{S C}$ the resonance peak is necessarily confined in both momentum and frequency space. We will show below that this situation is different in the underdoped compounds. Since $\Delta_{s w} \approx 2 \Delta_{S C}$ for $\mathrm{YBa}_{2} \mathrm{Cu}_{3} \mathrm{O}_{7}$, we expect $\Delta_{s w}>2 \Delta_{S C}$ for the magnetically overdoped compounds, where $\xi<2$, and no resonance peak should be observed (see inset in Fig. 11).

Fong et al. [8] find that $\omega_{\text {res }}$ increases slightly between $T_{c}$ and $T=10 \mathrm{~K}$. Since $\Delta_{s w}=c_{s w} / \xi$ in our model, their result implies that $\xi$ decreases over this temperature range, a finding consistent with the changes in the transverse relaxation rate, $T_{2 G}^{-1} \sim \xi$, in the superconducting state of $\mathrm{YBa}_{2} \mathrm{Cu}_{3} \mathrm{O}_{7}$ measured by Milling et al. 23] who report a decrease of $T_{2 G}^{-1}$ by about $10 \%$ with decreasing temperature. Put another way, in our model, the results of Milling et al. require that $\omega_{\text {res }}$ increase by some $10 \%$ below $T_{c}$.

The fact that $\omega_{\text {res }}$ is determined by the spin-gap $\Delta_{s w}$ explains the absence of the resonance peak in the even channel of $\mathrm{YBa}_{2} \mathrm{Cu}_{3} \mathrm{O}_{6+x}$. For $\mathrm{YBa}_{2} \mathrm{Cu}_{3} \mathrm{O}_{6.5}$, Bourges et al. [7] find a spin-gap, $\Delta_{s w}^{e v e n}=53, \mathrm{meV}$ in the even channel which may be expected to be larger in $\mathrm{YBa}_{2} \mathrm{Cu}_{3} \mathrm{O}_{7}$. Since $\Delta_{s w}^{e v e n}>\omega_{c}$ the damping of the even spin excitations around $\mathbf{Q}=(\pi, \pi)$ will not decrease upon entering the superconducting state and no resonance peak is to

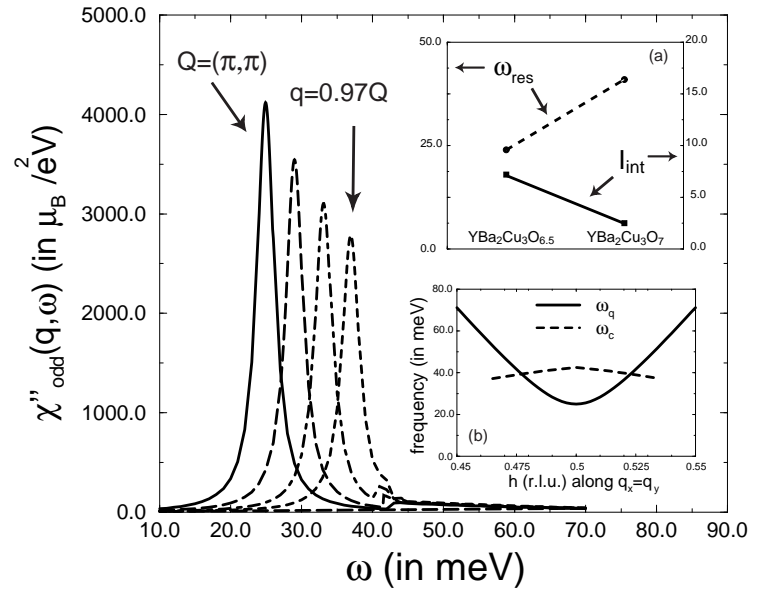

FIG. 3. The resonance peak for momenta in the vicinity of $\mathbf{Q}=(\pi, \pi)$. Inset $(a)$ : The doping dependence of $\omega_{\text {res }}$ (in meV, dashed line) and $\mathrm{I}_{\text {int }}$ (in $\mu_{B}^{2}$, solid line). Inset $(b)$ : Spin excitation spectrum and $\omega_{c}$ along $q_{x}=q_{y}$.

be expected.

We consider next the resonance peak in the underdoped $\mathrm{YBa}_{2} \mathrm{Cu}_{3} \mathrm{O}_{6+x}$ compounds; our results are summarized in inset $(a)$ of Fig. 3. Fong et al. find that $\omega_{\text {res }}$ decreases to $\omega_{\text {res }}=25 \mathrm{meV}$ in $\mathrm{YBa}_{2} \mathrm{Cu}_{3} \mathrm{O}_{6.5}$ while the integrated intensity of the peak increases from $\mathrm{I}_{\text {int }} \approx$ $1.1 \mu_{B}^{2}$ in $\mathrm{YBa}_{2} \mathrm{Cu}_{3} \mathrm{O}_{7}$ to $\mathrm{I}_{\text {int }} \approx 2.6 \mu_{B}^{2}$ in $\mathrm{YBa}_{2} \mathrm{Cu}_{3} \mathrm{O}_{6.5}$. In our model, the doping dependence of $\omega_{\text {res }}=\Delta_{s w}=$ $c_{s w} / \xi$ is determined by changes in $c_{s w}$ and $\xi$. From an analysis of NMR [20] and INS experiments [19], we know that the correlation length at $T_{c}$ increases from $\xi \approx 2.2$ in $\mathrm{YBa}_{2} \mathrm{Cu}_{3} \mathrm{O}_{7}$ to $\xi \approx 6$ in $\mathrm{YBa}_{2} \mathrm{Cu}_{3} \mathrm{O}_{6.5}$. This change in $\xi$ more than compensates any increase of $c_{s w}$ as the doping is reduced and so brings about a decrease in $\Delta_{s w}$. Furthermore, Bourges et al. [7] find a normal state spingap in $\mathrm{YBa}_{2} \mathrm{Cu}_{3} \mathrm{O}_{6.5}, \Delta_{s w} \approx 23 \mathrm{meV}$ [7], in agreement with the position of the resonance peak in the superconducting state reported by Fong et al. 便. The integrated intensity $\mathrm{I}_{\text {int }}$ of the resonance peak in the limit $\operatorname{Im} \Pi_{o d d} \ll 1 / \alpha \xi^{2}$, i.e., for small spin damping, is given by $\mathrm{I}_{\text {int }}=\alpha \xi^{2} \Delta_{s w} \pi / 4$. For $\mathrm{YBa}_{2} \mathrm{Cu}_{3} \mathrm{O}_{7}, \alpha=15 \mu_{B}^{2} / \mathrm{eV}$ which yields $\mathrm{I}_{\text {int }}=(2.3 \pm 0.4) \mu_{B}^{2}$. For $\mathrm{YBa}_{2} \mathrm{Cu}_{3} \mathrm{O}_{6.5}$, we find from our analysis of INS data in the normal state $\alpha \approx 9 \mu_{B}^{2} / e V$, and thus $\mathrm{I}_{\text {int }}=(6.2 \pm 1.0) \mu_{B}^{2}$. Both values for $\mathrm{I}_{\text {int }}$ are a factor of 2 larger than the values measured experimentally by Keimer et al. [5]; however, the relative increase of $I_{\text {int }}$ on going from $\mathrm{YBa}_{2} \mathrm{Cu}_{3} \mathrm{O}_{7}$ to $\mathrm{YBa}_{2} \mathrm{Cu}_{3} \mathrm{O}_{6.5}$ is in quantitative agreement with their results.

As $2 \Delta_{S C}$ and $\Delta_{s w}$ become well separated in the underdoped compounds 14, 15, we predict a resonance peak for all momenta in a region around $\mathbf{Q}$ which is determined by $\omega_{q}<\omega_{c}$ (see inset (b) of Fig. [3). In Fig. 3 we show how the resonance peak shifts to higher frequencies when one moves in momentum space from $\mathbf{Q}=(\pi, \pi)$ to 
$\mathbf{q}=0.97(\pi, \pi)$. Due to the limited momentum resolution of INS experiments we do not expect that these peaks can be resolved; this implies that one should only observe one broad peak which extends from $\Delta_{s w}$ up to $\omega_{c}$. This broadening of the peak width is just what is observed by

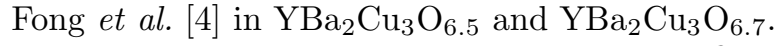

For the underdoped compounds, two groups find a precursor of the resonance peak in the normal state below $T_{*} \approx 200 \mathrm{~K}$, in the so-called strong pseudo-gap region [4, 1 ]. At the same time, ARPES experiments find that below $T_{*}$, the low-frequency spectral weight of the "hot" fermionic quasi-particles around $(0, \pi)$ is suppressed (the "leading-edge gap") [14], which immediately leads to a decrease of the spin-damping at $\mathbf{Q}=(\pi, \pi)$ and an enhancement of $\chi_{o d d}^{\prime \prime}$. Our model thus predicts an enhancement of $\chi_{o d d}^{\prime \prime}$ around $\omega_{\text {res }}$ in the normal state of those superconductors for which a leading edge gap occurs below $T_{*}$.

It follows from the above analysis, that a resonance peak should occur in all cuprate superconductors for which the condition $\Delta_{s w}<2 \Delta_{S C}$ is satisfied. This implies that the resonance peak is not directly related to the bilayer structure of $\mathrm{YBa}_{2} \mathrm{Cu}_{3} \mathrm{O}_{6+x}$. In $\mathrm{La}_{2-x} \mathrm{Sr}_{x} \mathrm{CuO}_{4}$, where $\Delta_{S C} \approx 9 \mathrm{meV}$ and $\Delta_{s w}>25 \mathrm{meV}$ [24], the above condition is not satisfied and consequently, no resonance peak is to be expected. On the other hand, for the single-layer material $\mathrm{HgBa}_{2} \mathrm{CuO}_{4+\delta}$ with an optimum $T_{c}=95 \mathrm{~K}$, we expect $\Delta_{S C}$ to be comparable to that found in $\mathrm{YBa}_{2} \mathrm{Cu}_{3} \mathrm{O}_{7}$, so that it is a good candidate for the observation of a resonance peak.

Our model also provides a natural explanation for the results of recent INS experiments on Zn-doped $\mathrm{YBa}_{2} \mathrm{Cu}_{3} \mathrm{O}_{6.97}$ [25]. A Zn concentration of $2 \%$ which suppresses $T_{c}$ to $T_{c}=69 \mathrm{~K}$ completely destroys the resonance peak in the superconducting state, and generates a significant amount of spectral weight in $\chi^{\prime \prime}$ at low frequencies. Since $\Delta_{s w} \approx 2 \Delta_{S C}$ in this material, to the extent that $\Delta_{s w}$ is not markedly influenced by $\mathrm{Zn}$, a quite modest Zn-induced decrease in $\Delta_{S C}$ will render the resonance unobservable in the superconducting state. For the underdoped compounds, on the other hand, since $\Delta_{s w}$ and $2 \Delta_{S C}$ are more clearly separated, a larger amount of $\mathrm{Zn}$ would be required to suppress the resonance. The appearance of low-frequency spectral weight in $\chi^{\prime \prime}$ shows that a considerable spin-damping persists in the superconducting state, as might be expected from Zn-induced changes in the low-frequency spectral weight in the single-particle spectrum.

In summary we have shown that a spin-wave model provides a natural explanation for the doping and temperature dependence of the existing INS experiments on the appearance of a resonance peak in the superconducting state and of a resonance feature in the normal state of underdoped systems. We proposed necessary conditions for the observation of the peaks and resonance features, and so explain the failure to observe either of them in $\mathrm{La}_{2-x} \mathrm{Sr}_{x} \mathrm{CuO}_{4}$. We argue that the occurrence of the resonance peak is not directly related to the bilayer struc- ture of $\mathrm{YBa}_{2} \mathrm{Cu}_{3} \mathrm{O}_{6+x}$, and predict that it should also be observable in single-layer cuprates with a sufficiently large superconducting gap.

We would like to thank P. Bourges, A.V. Chubukov, P. Dai, T. Fong, B. Keimer, B. Lake, T. Mason, A. Millis, H. Mook, and J. Schmalian for valuable discussions. This work has been supported in part by the Science and Technology Center for Superconductivity through NSFgrant DMR91-20000.

[1] J. Rossat-Mignod et al. Physica C 185-189, 86 (1991); H. A. Mook et al. Phys. Rev. Lett. 70, 3490 (1993); H.F. Fong et al., Phys. Rev. Lett. 75, 316 (1995); P. Bourges et al. Phys. Rev. B 53, 876 (1996).

[2] P. Dai et al., Phys. Rev. Lett. 77, 5425 (1996).

[3] P. Dai et al.,Phys. Rev. Lett. 80, 1738 (1998).

[4] H.F. Fong et al., Phys. Rev. Lett. 78, 713 (1997).

[5] B. Keimer et al., preprint, cond-mat 9705103 .

[6] S. Hayden et al., preprint, cond-mat 9710181.

[7] P. Bourges et al., Phys. Rev. B 56, R11439 (1997); P. Bourges, preprint.

[8] H.F. Fong et al. Phys. Rev. B 54, 6708 (1996).

[9] I.I. Mazin and V.M. Yakovenko, Phys. Rev. Lett. 75, 4134 (1995); D.Z. Liu, Y. Zha, and K. Levin, Phys. Rev. Lett. 75, 4130 (1995); A. Millis and H. Monien, Phys. Rev. B 54, 16172 (1996).

[10] N. Bulut and D. Scalapino, Phys. Rev. B 53, 5149 (1996); G. Blumberg, B.P. Stojkovic, and M.V. Klein, Phys. Rev. B 52, R15741 (1996).

[11] L. Yin, S. Chakravarty, P.W. Anderson, Phys. Rev. Lett. 78, 3559 (1997).

[12] E. Demler and S. C. Zhang, Phys. Rev. Lett. 75, 4126 (1995); J. Brinckmann and P. A. Lee, preprint, cond-mat 9710065 .

[13] V. Barzykin and D. Pines, Phys. Rev. B 52, 13585 (1995).

[14] A.G. Loeser et al., Science 273, 325 (1996); H. Ding et al., Nature 382, 51 (1996).

[15] Ch. Renner et al., Phys. Rev. Lett. 80, 149 (1998).

[16] The exact value of $\omega_{c} \approx 2 \Delta_{S C}$ depends on bandstructure parameters; since it is not important for our scenario, for simplicity we take $\omega_{c}=2 \Delta_{S C}$.

[17] P. Monthoux and D. Pines, Phys. Rev B 47, 6069 (1993).

[18] A.V. Chubukov, S. Sachdev, and J. Ye, Phys. Rev. B 49, 11919 (1994).

[19] D.K. Morr and D. Pines, in preparation.

[20] Y. Zha, V. Barzykin and D. Pines, Phys. Rev. B 54, 7561 (1996).

[21] I. Maggio-Aprile et al, J. Low Temp. Phys. 105, 1129 (1996).

[22] A.V. Chubukov and D.K. Morr, Phys. Rep. 288, 355 (1997).

[23] C. Milling, private communication.

[24] T. Mason, private communication.

[25] Y. Sidis et al., Phys. Rev B 53, 6811 (1996). 